\title{
EDITORIAL
}

\section{Thank you to our reviewers!}

\author{
Mucosal Immunology (2019) 12:293-295; https://doi.org/10.1038/s41385-019-0142-8
}

Editor-in-chief

The editors at Mucosal Immunology would like to extend their heartfelt thanks to those who have provided their time and energy to review manuscripts for them over the 2018 calendar year. As you are well aware, we take the review process very seriously, and your concerted efforts to provide concise, accurate, and thoughtful reviews benefitted not only the quality of the articles we publish, but also the authors, who gained insight and guidance to make their manuscript more convincing and impactful. Furthermore, your valuable feedback and discussions of contested reviews (of which there were few) were, and are invaluable in developing new ideas to improve what will always be an imperfect process. In this regard, in the near future you will see meaningful changes in our on-line review forms, and we are developing better resources to guide less experienced reviewers. We should also celebrate the fact of 626 completed reviews in 2018, the average time from reviewer assignment to completion was 14 days, a milestone that we have been seeking to achieve. Below is a list of all of you who completed at least one review, and we give special recognition $\left(^{*}\right)$ to those who provided three or more. As always, we are open to your thoughts on ways to improve our review process, and welcome ideas for exciting new areas we should address with reviews and commentaries as we move forward into our twelfth year in press!

\author{
Abdel-Mohsen, Mohamed \\ Abraham, Soman \\ Actor, Jeffrey K. \\ Akbari, Omid \\ Alcorn, John \\ Allen, Irving \\ Ancuta, Petronela \\ Arditi, Moshe \\ Artis, David \\ Asehnoune, Karim \\ Atreya, Raja \\ Ayabe, Tokiyoshi \\ Azcutia, Veronica* \\ Bailey, Michael T. \\ Bailey, Mick \\ Bain, Calum \\ Ballesteros-Tato, Andre \\ Barber, Daniel \\ Barrett, Terry \\ Bayry, Jagadeesh \\ Beisswenger, Christoph \\ Bemark, Mats* \\ Benoist, Christophe \\ Berg, Leslie \\ Berin, Cecilia \\ Berlec, Ales \\ Bertin, Benjamin \\ Berwin, Brent \\ Bhattacharya, Jahar \\ Bilsborough, Janine \\ Bingle, Colin \\ Blaser, Martin J. \\ Boirivant, Monica \\ Bont, Louis \\ Bontkes, Hetty \\ Borish, Larry \\ Boritz, Eli \\ Bose, Tanima \\ Bosinger, Steven \\ Bouchery, Tiffany \\ Boyaka, Prosper
}

Bozinovski, Steven

Braciale, Thomas J.

Brandt, Curtis R.

Braun, Jonathan

Brazil, Jennifer

Brenchley, Jason

Britton, Warwick

Brown, Andrew

Brownlie, Rebecca

Bryceson, Yenan T.

Budelsky, Alison

Burgener, Adam

Burwitz, Benjamin

Bustamante, Jacinta

Byrne, Adam

Camp, Jeremy

Cannon, Martin J.

Carlin, Leo

Carr, Daniel

Carvalho, Frederic

Caspi, Rachel

Caucheteux, Stephan

Cauley, Linda

Cerf-Bensussan, Nadine

Cerovic, Vuk

Cerutti, Andrea

Chamoto, Kenji

Chan, John

Chang, Theresa

Chassaing, Benoit

Chatila, Talal

Chen, Grace

Chen, Kong

Chevalier, Gregoire

Chiu, Chris*

Chong, Shu Zhen

Chroneos, Zissis C.

Chung, Doo Hyun

Clarke, Thomas

Coburn, Lori

Cohn, Lillian

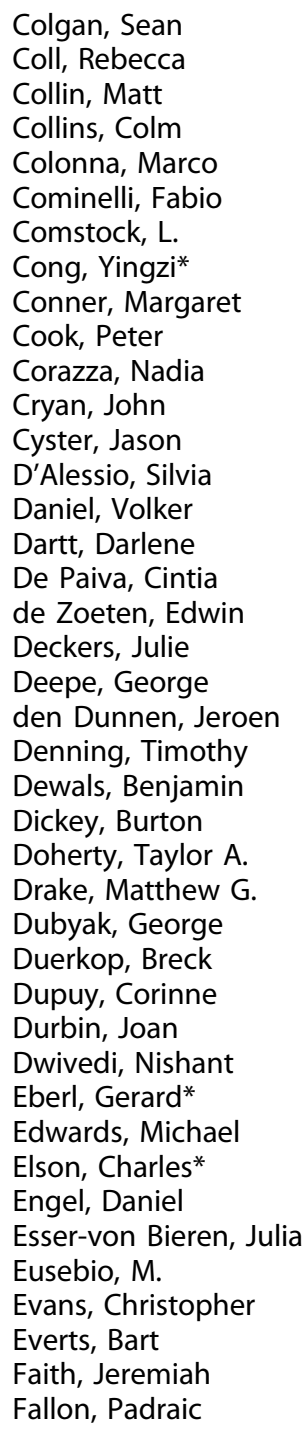

Farber, Donna

Fehrenbach, Heinz

Feng, Carl

Fernandez-Ruiz, Daniel

Ferreira, Daniela

Filardy, Alessandra

Fiocchi, Claudio

Foronjy, Robert

Forsyth, Christopher

Forthal, Donald

Fox, James

Franke, Andre

Freud, Aharon

Fukui, Hirokazu

Fulde, Marcus

Gabel, Chris

Gadjeva, Mihaela

Ganal-Vonarburg, Stephanie

Gasteiger, Georg

Gause, William

Genescà, Meritxell

Georas, Steven

Gerber, Anthony

Geremia, Alessandra

Gershon, Michael

Gershwin, Merrill

Gewirtz, Andrew

Giannoni, Eric

Gilbert, Sarah

Girard, Jean-Phillippe

Godfrey, Dale

Gosens, Reinoud

Gottschalk, Marcelo

Grainger, John

Grasberger, Helmut

Grimbacher, Bodo

Guida, Giuseppe

Guillot, Loic

Günther, Claudia

Guo, Xiaohuan*

Gurish, Michael 
Gutierrez, Maximiliano

Hackam, David

Haigwood, Nancy

Hajishengallis, George

Hall, Pamela R.

Halle, Stephan

Haller, Dirk

Hammarstrom, Lennart

Hanson, Julien

Hansson, Gunnar

Harandi, Ali

Harker, James*

Hase, Koji

Hashiguchi, Masaaki

Haspel, Jeffrey

Heijink, Irene*

Heinemann, Akos

Hendricks, Robert L.

Hendriks, Rudolf

Henrich, Timothy

Hepworth, M.

Herzog, Erica

Hessell, Ann

Hewitson, James

Heymann, Felix

Hiemstra, Pieter

Hodge, Greg

Holt, Patrick

Hornef, Mathias

Horsnell, William

Huckriede, Anke

Huffnagel, Gary

Hussell, Tracy

Imai, Yasuyuki

Ishihara, Shunji

Ivanov, Andrei*

lyer, Smita

Izcue, Ana

Jacques, Yannick

Jakubzick, Claudia

Jameson, Stephen

Kamada, Nobuhiko*

Kanai, Takanori

Kanaya, Takashi

Kanellopoulos, Colette

Kashyap, Purna C.

Kaul, Rupert*

Kayama, Hisako

Keane, Joseph

Kedzierska, K.

Kent, Stephen

Keshavazian, Ali

Khader, Shabaana

Khan, Imtiaz

Khanna, Kamal

Kiela, Pawel

Kim, Chang*

Kim, Hye-Young

Kirman, Joanna

Kitani, Atsushi

Klase, Zachary

Klein, Bruce

Koch, Stefan

Kolls, Jay*

Konkel, Joanne

Konnikova, Liza

Kool, Mirjam

Koropatkin, Nicole
Kousoulas, K. Gus

Kowalczyk, Andrew

Krammer, Florian

Krausgruber, Thomas

Kweon, Mi-Na

Lahl, Katharina

Landay, Alan

Le Gros, Graham

Lee, Gap Ryol

Lee, Myung-Shik

Legge, Kevin*

Lelouard, Hugues

Lencer, Wayne

Lewinsohn, David

Lewis, Michael D.

Lewkowich, Ian

Lindenstrom, Thomas

Linterman, Michelle

Liu, Zhanju

Lochner, Matthias

Loke, P'ng

Longman, Randy

Loré, Karin

Loxton, Andre G.

Lu, Ben

Lu, Li-Fan

Lubberts, Erik

Mabbott, Neil

MacDonald, Andrew

MacDonald, Tom

Macnaughton, Wallace

Mahe, Maxime

Maile, Robert

Maizels, Rick M.

Malarkannan, Subramaniam

Malin, Stephen

Mallevaey, Thierry

Maloy, Kevin

Mann, Elizabeth*

Mannon, Peter

Mantis, Nicholas

Marino, Eliana

Marrazzo, Jeanne

Marsland, Benjamin

Martin, Filar

Martinelli, Elena

Marvel, Jacqueline

Maslowski, Kendle

Masson, Lindi

Masters, Seth

Masterson, Joanne

Matteoli, Gianluca

Maxwell, Joe*

Mays, Jackie

McCluskey, James

McCole, Declan

McCormick, Beth

McCoy, Kathy D.

McCusker, Christine

McGuckin, Michael*

McKay, Derek

McKinnon, Lyle

McNagny, Kelly

McNamee, Eoin

McSorley, Henry

Mempel, Thorsten

Menard, Sandrine

Meresse, Bertrand*
Mestecky, Jiri

Miller, Mark

Milligan, Gregg

Milling, Simon*

Mills, Kingston

Mitchell, Caroline

Mizoguchi, Atsushi*

Mjosberg, Jenny*

Mohan, Mahesh

Montaner, Luis

Monteleone, Giovanni

Mora, J. Rodrigo

Mortha, Arthur

Mowat, Allan*

Mucida, Daniel

Mueller, Anne

Mueller, Christoph

Murphy, Kenneth

Nagaishi, Takashi

Nakao, Atsuhito

Namasivayam, Sivaranjani

Nardelli-Haefliger, Denise

Nawijn, Martijn

Nayak, Jayakar

Neish, Andrew

Neurath, Markus

Niederkorn, Jerry

Niess, Jan*

Nieuwenhuis, Edward

Noursadeghi, Mahdad

Nowak, Piotr

Nunez, Gabriel

Nusrat, Asma

Nutman, Thomas

Nutt, Stephen L.

Ohno, Hiroshi

Okamoto, Yoshitaka

Olivares-Villagómez, Danyvid

Oliver, Brian G. G.

Orend, Gertraud

Ortiz, Alexandra

Osorio, Manuel

Ota, Yuk

Ouellette, Andre

Overstreet, Anne-Marie

Pabst, Oliver

Paclet, Marie Hélène

Paiardini, Mirko*

Palm, Noah

Palmer, Brent E.

Pasetti, Marcela

Patel, Mickey

Paul, Stephane

Pearson, Claire

Peebles, Stokes

Pekosz, Andrew

Peng, Sui

Pepper, Marion

Permar, Sallie

Petronilho, Fabricia

Petrovas, Costas

Philpott, Dana

Pickard, Joseph

Pinelli, Elena

Poholek, Amanda

Porter, Joanna

Pothoulakis, Charalabos

Pott, Johanna
Prodger, Jessica

Provine, Nicholas

Prussin, Calman

Pütsep, Katrin

Qiao, Shuo-Wang

Qin, Chengyong

Quesniaux, Valérie

Quiros, Miguel

Raffatellu, Manuela

Raghavan, Sukanya

Ray, Anuradha

Reeves, Keith

Reeves, R. Keith

Reinhardt, Christoph

Rengarajan, Jyothi

Rivera, Amariliz

Roan, Nadia

Robert-Guroff, Marjorie

Robinson, Keven

Rodriguez-Garcia, Marta

Ronchese, Franca

Roxby, Alison C.

Ruckwardt, Tracy

Ruemmele, Frank

Russell, Michael

Saban, Daniel

Saglani, Sejal*

Saito, Tatsuya

Sajjan, Uma

Salek-Ardakani, Shahram

Samsom, Janneke

Sandberg, Johan

Sander, Leif-Erik

Santra, Sampa

Saunders, Bernadette

Sawa, Shinichiro

Schippers, Angela

Schlegel, Nicolas

Schneider, K. M.

Schulzke, Joerg

Scott, Charlotte

Sheridan, Brian

Shin, Sunny

Shore, Stephanie

Siegmund, Britta*

Silbart, Larry

Silva-Santos, Bruno

Singh, Vishal

Skalski, Joseph

Smith, Amber M.

Smith, Kelly

Smythies, Lesley

Sonnenberg, Gregory*

Spencer, Jo

Spits, Hergen

St. Leger, Anthony

Stagg, Andy

Stampfli, Martin

Stegemann-Koniszewski, Sabine

Strauss, Gudrun

Sundrud, Mark

Suratt, Benjamin

Sutterwala, Fayyaz

Sutton, Philip

Svensson, Mattias

Tacchini-Cottier, Fabienne

Takeda, Kiyoshi 
Tanaka, Kazuo

Tarleton, Rick

Tartour, E.

Taylor, Andrew

Teluguakula, $\mathrm{N}$

Tenbusch, Matthias

Teshima, Takanori

Thimmulappa, Rajesh K.

Tobin, David

Togashi, Yosuke

Torow, Natalia

Toubai, Tomomi

Towne, Jennifer*

Tripathi, Deepak

Trottein, Francois

Turnquist, Heth R.

Tussiwand, Roxane

Uematsu, Satoshi
Underhill, D.

Unutmaz, Derya

Upadhyay, Chitra

Upham, John

Vacca, Paola

Vaccari, Monica

Valatas, Vassilis

Van Den Steen, Philippe

Varga, Steven

Velin, Dominique

Ventecleft, Nicolas

Vezys, Vaiva

Vijay-Kumar, Matam

Viney, Jo

Vocanson, Marc

Vossenkamper, Anna

Wakim, Linda

Walmsley, Sarah
Wang, De-Yun

Watanabe, Tomohiro

Whitney, James

Wick, Mary Jo

Willinger, Tim

Wilson, Mark

Winter, Nathalie

Winter, Sebastian

Withers, David

Worthington, John

William Agace ${ }^{1}$, Nadine Cerf-Bensussan ${ }^{2}$,
Clare Lloyd ${ }^{3}$ and Brian Kelsall
${ }^{4}$
${ }^{1}$ Lund University, Lund, Sweden; ${ }^{2}$ Université Paris Descartes-Sorbonne,
Paris, France; ${ }^{3}$ Imperial College, London, United Kingdom and
${ }^{4}$ National Institutes of Health, Bethesda, MD, USA
Correspondence: Brian Kelsall (bkelsall@gmail.com)

William Agace ${ }^{1}$, Nadine Cerf-Bensussan ${ }^{2}$,
Clare Lloyd ${ }^{3}$ and Brian Kelsall
${ }^{4}$
${ }^{1}$ Lund University, Lund, Sweden; ${ }^{2}$ Université Paris Descartes-Sorbonne,
Paris, France; ${ }^{3}$ Imperial College, London, United Kingdom and
${ }^{4}$ National Institutes of Health, Bethesda, MD, USA
Correspondence: Brian Kelsall (bkelsall@gmail.com)

William Agace ${ }^{1}$, Nadine Cerf-Bensussan ${ }^{2}$,
Clare Lloyd ${ }^{3}$ and Brian Kelsall

William Agace ${ }^{1}$, Nadine Cerf-Bensussan ${ }^{2}$,
Clare Lloyd ${ }^{3}$ and Brian Kelsall
${ }^{4}$
${ }^{1}$ Lund University, Lund, Sweden; ${ }^{2}$ Université Paris Descartes-Sorbonne,
Paris, France; ${ }^{3}$ Imperial College, London, United Kingdom and
${ }^{4}$ National Institutes of Health, Bethesda, MD, USA
Correspondence: Brian Kelsall (bkelsall@gmail.com)

William Agace ${ }^{1}$, Nadine Cerf-Bensussan ${ }^{2}$,
Clare Lloyd ${ }^{3}$ and Brian Kelsall
${ }^{4}$
${ }^{1}$ Lund University, Lund, Sweden; ${ }^{2}$ Université Paris Descartes-Sorbonne,
Paris, France; ${ }^{3}$ Imperial College, London, United Kingdom and
${ }^{4}$ National Institutes of Health, Bethesda, MD, USA
Correspondence: Brian Kelsall (bkelsall@gmail.com)

William Agace ${ }^{1}$, Nadine Cerf-Bensussan ${ }^{2}$,
Clare Lloyd ${ }^{3}$ and Brian Kelsall
${ }^{4}$
${ }^{1}$ Lund University, Lund, Sweden; ${ }^{2}$ Université Paris Descartes-Sorbonne,
Paris, France; ${ }^{3}$ Imperial College, London, United Kingdom and
${ }^{4}$ National Institutes of Health, Bethesda, MD, USA
Correspondence: Brian Kelsall (bkelsall@gmail.com)

Wright, Terry

Xiang, Zhou

Xing, Zhou

Yang, Xuexian

Zenewicz, Lauren

Zuohua

Zheng, Tim

Zhou, Xuyu

Zilinski, Christina

Zlotnik, Albert 